\title{
Del poder duro al poder inteligente. La nueva estrategia de seguridad de Barack Obama o de la sobrevivencia de la política exterior de Estados Unidos*
}

\author{
José Luis VALDÉs-UGALDE ${ }^{* *}$ \\ FRANIA DUARTE ${ }^{* * *}$
}

\section{RESUMEN}

El uso del poder duro y la negligente política económica y política de la administración Bush provocaron una crisis de legitimidad en el liderazgo internacional estadunidense. Para enmendar esta situación, Obama optó por aplicar la política de poder inteligente ("smart power"); sin embargo, en ocasiones su puesta en marcha ha sido obstaculizada por la polarización política y la crisis económica internas. A pesar de las circunstancias, dicha política ha mostrado ser viable para lograr los objetivos de la política exterior de Estados Unidos, toda vez que, frente a diversos sucesos, ha permitido recuperar gradualmente algunos de los espacios de influencia en el mundo perdidos por Estados Unidos.

Palabras clave: Barack Obama, hegemonía, declive, política exterior, poder inteligente, seguridad.

\section{ABSTRACT}

The Bush administration's use of hard power and negligent economic and political policy caused a crisis of legitimacy of U.S. international leadership. To set this situation right, Obama opted for a smart power policy. However, its implementation has sometimes been blocked by domestic political polarization and economic crisis. Despite the circumstances, this policy has shown itself to be viable for achieving U.S. foreign policy aims since, in the face of different events, it has made it possible to gradually recover some of the spaces of influence in the world that the United States had lost.

Key words: Barack Obama, hegemony and decline, foreign policy, smart power, security.

* Este trabajo se realizó durante una estancia sabática en la Freie Universität Berlin por el periodo 2013-2015. Agradezco a la DGAPA, UNAM su apoyo en la realización del mismo.

** Ex director (2001-2009) e investigador del cisan, unAm. Profesor de la Facultad de Ciencias Políticas y Sociales (ғсPys), UNAM. Investigador y profesor visitante en la Freie Universität Berlin por el periodo 2013-2015, jlvaldes@unam.mx y jvaldes@zedat.fu-berlin.de

*** Licenciada en Relaciones Internacionales por la FCPys, UNAM. Profesora adjunta en la FCPys, UnAM, fzduartt @gmail.com 


\section{INTRODUCCIÓN}

The pressure of the State machine is nothing compared with the pressure of a convincing argument.

Czeslaw Milosz

La política exterior de la administración Obama ha encontrado diversas dificultades para ser ejecutada. En primer lugar, el ejercicio del poder duro de G.W. Bush condujo a la pérdida de legitimidad internacional de Estados Unidos, lo cual, a su vez, produjo un elevado gasto de defensa que coadyuvó al incremento del déficit presupuestal y de la deuda pública. En segundo lugar, la negligencia ante la política económica y financiera derivó en la crisis de 2008, como la etapa crítica y más reciente de una larga crisis económica que Estados Unidos ya arrastraba. Por otro lado, el reciente descubrimiento del espionaje estadunidense a varios de sus socios en el mundo, así como la crisis en Siria, han puesto en riesgo la política de poder inteligente (smart power).

Todo esto pone a Obama en una difícil situación en la que las decisiones deben tomarse con cautela y con el mayor dinamismo posible, antes de que las crisis, dentro y fuera de Estados Unidos, se agraven más. A nivel interno, Obama enfrenta a una población expuesta al desempleo (el 7.3 por ciento), al tiempo que los sectores ultraconservadores en el Congreso han rechazado las iniciativas que él ha formulado para revertir los efectos de la crisis. A nivel externo, Obama ha tenido que replantear la estrategia de política exterior y ocuparse de los asuntos que son vitales para que Estados Unidos recupere su posición como actor predominante en el concierto internacional y en el cual nuevos actores están emergiendo y se perfilan como importantes tomadores de decisiones.

En este contexto, han surgido interrogantes cruciales, en cuyas respuestas Estados Unidos busca mantener su posicionamiento como actor preponderante en el medio internacional, característica que coadyuva a alimentar parcialmente su poderío nacional: ¿cómo lograr un equilibrio para abordar asuntos de política interna y externa, toda vez que ésta no es de vital interés para los ciudadanos estadunidenses en el contexto de la crisis económica?, ¿cómo ejercer una política exterior que coadyuve a avanzar en los intereses y objetivos de Estados Unidos en el mundo, pero sin que eso propicie condiciones que provoquen un mayor sentimiento antiestadunidense?, ¿cómo construir una política exterior que no sólo se apegue a los objetivos propuestos, sino que además se adapte a los recortes presupuestales en materia de seguridad y defensa? 
En respuesta a dichos planteamientos, Obama ha decidido aplicar la estrategia de smart power. En el marco de esta propuesta, intenta concretar los intereses de Estados Unidos en el mundo mostrando una nueva cara: la de un país comprometido con el bien común y la defensa de los bienes globales, y con amplia disposición al diálogo (poder suave), dejando como último recurso el uso de la fuerza (poder duro). Se favorece, entonces, una política de carácter preventivo y contencionista.

A pesar de que en el camino ha habido obstáculos para su aplicación, el desarrollo de esta estrategia parece reportar más beneficios que desventajas para los intereses estadunidenses. De hecho, por lo que señaló en su último discurso sobre el estado de la Unión, es evidente que Obama ha tomado en cuenta que la estrategia de poder inteligente ha coadyuvado (y lo podría seguir haciendo), a pesar de las limitaciones prácticas que ha enfrentado en el terreno, a alcanzar el equilibrio que la opinión pública desea ver entre la política interna y la externa, así como el equilibrio a nivel internacional que se esperaba desde antes de 2008 frente a los actores del concierto internacional. ${ }^{1}$

\section{LA POLÍTICA EXTERIOR ESTADUNIDENSE COMO MEDIO PARA LA CONSERVACIÓN DE LA SUPREMACÍA INTERNACIONAL}

La política exterior de los Estados se define en torno a las necesidades, capacidades e intereses de cada actor, así como por su contexto interno y externo. Uno de los intereses de los Estados es lograr un entorno de seguridad, definida por Buzan (1992: 432) como "la búsqueda de la libertad de las amenazas y la habilidad de los Estados y sociedades para mantener su identidad independiente y su integridad funcional contra las fuerzas de cambio que perciben como hostiles". Así, dentro de un entorno seguro, cada Estado podrá idealmente cumplir sus objetivos; no obstante, el proceso de definición de un entorno de seguridad es subjetivo, de tal manera que lo que es seguro para un Estado podría no serlo para otro y viceversa.

En el caso de Estados Unidos, ese entorno seguro no se limita a la protección de sus fronteras territoriales, sino que las trasciende. Esto ocurre en medio de un fenómeno en el que las fronteras han dejado de definirse solamente en términos territoriales, dando lugar a las fronteras no territoriales (Cuttita, 2006). Así, las fronteras se extienden y transforman debido al proceso de mapeo del mundo, dando lugar a la

\footnotetext{
${ }^{1}$ Cabe señalar, no obstante, que en materia de política exterior hubo fuertes divergencias entre Hillary Clinton y Barack Obama, que al final fueron desdibujándose, al adherirse Clinton al gobierno de Obama como secretaria de Estado e incorporar en su agenda muchas de las propuestas que fueron motivo de polémica durante las primarias (véase Mann, 2012).
} 
redefinición multidimensional de los diversos tipos de fronteras: económicas, sociales, culturales, lingüísticas, geopolíticas, etc., por lo que se abarcan diversas regiones del globo y, por ende, diversos actores y temas. Este fenómeno de extensión de fronteras no le ocurre a Estados Unidos solamente debido a que el proceso de globalización así lo demande, sino porque la política de seguridad estadunidense gira en función de, entre otras cosas, mantener su posición como actor preponderante del mundo global. De ahí que todo asunto que afecte el carácter transnacional de su influencia siempre tiene como prioridad aquello que marca la gran estrategia en política internacional, a la cual seguirá respondiendo proactivamente.

Cabe señalar que el interés estadunidense por mantener esta postura no es reciente, sino que ha existido desde antes de su fundación como nación moderna, cuando los primeros colonos ingleses llegaron al continente americano, compartiendo la idea de John Winthrop de fundar una "ciudad sobre la colina", idea que por cierto no devino exclusivamente de las expectativas que el potencial del territorio privilegiado al cual arribaron les producía, sino de la creencia puritana de haber sido elegidos por la divinidad para civilizar al mundo. En pocas palabras, se trataba de las ideas contenidas en el Destino Manifiesto, que posteriormente se complementaron con, primeramente, el carácter de nación excepcional, toda vez que se estimaba que Estados Unidos había nacido como una democracia "pura", y en virtud de que no había tenido pasado feudal ni transitado por un proceso revolucionario y, en segundo lugar, de la promoción del credo americano, es decir, la de los valores universales de libertad, igualdad y justicia, así como de la democracia y el libre mercado. ${ }^{2}$

De tal forma, el afán de mantener el statu quo en el mundo dicta el quehacer de la política exterior de Estados Unidos, que en su papel de actor aún predominante produce e impone las condiciones necesarias para que esta condición se mantenga. Para ello es necesario el empleo y optimización de los recursos y las capacidades con que el Estado cuente, todo lo cual se traduce en el ejercicio del poder nacional y, más específicamente, en el ejercicio del poder duro de coerción o del poder suave de atracción o de la combinación de ambos expresada en el poder inteligente (Nye, 2011).

\section{La ADMINISTRACIÓN BUSH Y "EL SILENCIO DEL CENTRO RACIONAL"}

Cada vez que un presidente estadunidense inicia su mandato asume, entre otras, la responsabilidad de mantener condiciones favorables de ejercicio del poder vis-à-vis

\footnotetext{
${ }^{2}$ El tema del excepcionalismo de Estados Unidos es de enorme vigencia en la actualidad y ciertamente ha empezado a desmitificarse y a ser revisado por historiadores y politólogos estadunidenses, como Stephen M. Walt, Thomas L. Friedman y Michael Mandelbaum (véase Foreign Policy, 2011).
} 
la necesidad de conservar el statu quo global. Cuando George W. Bush llegó a la presidencia acompañado de la segunda generación de neoconservadores en el poder (Velasco Grajales, 2010) consideró que la mejor manera de conservar la supremacía estadunidense en el mundo era a través de la fuerza (poder duro).

Una vez ocurridos los atentados terroristas del 11 de septiembre de 2001, la guerra contra el terrorismo global y la doctrina Bush, o doctrina de la acción anticipatoria (preemptive action), cedieron el paso al poder duro, primero en Afganistán y posteriormente en Irak. El mundo quedó inmerso en el marco del diseño geopolítico estadunidense, trazado a partir de la necesidad de combatir a la nueva amenaza terrorista (no estatal) que, aunque movible, siempre fue asociada con un agente fijo (estatal), por lo que durante el proceso del remapeo de sus prioridades globales, Estados Unidos fijó nuevas fronteras de seguridad que separaban a quienes apoyaban la guerra contra el terrorismo, de los que apoyaban a los terroristas. En estos últimos confines quedaron atrapados Irak, Irán y Corea del Norte, denominados por Bush como el "eje del mal".

No obstante, hubo un obstáculo por el cual esa política exterior no produjo durante la era de Bush los efectos deseados (capturar a Osama bin Laden, derrotar a Al-Qaeda y a los talibán, así como lograr el predominio mundial) sino que, por el contrario, condujo a Washington a una crisis de liderazgo internacional. Ese obstáculo radicó en que la política exterior se desarrolló en el contexto de lo que Halper y Clarke (2007) denominan "el silencio del centro racional". Ante los excesos de la elite política neoconservadora que se condujo con desparpajo y temeridad, en el nombre de un mesianismo de otro tiempo, se arrastró al establecimiento de política exterior a una actuación que se distinguió por la satisfacción de intereses personales y especiales, sin considerar los efectos que la vuelta a los valores más recalcitrantes del credo estadunidense ocasionaría a Estados Unidos. El "silencio" del centro racional de decisiones no solamente se produjo ante el enmudecimiento de los neoconservadores y de otros sectores de las elites políticas por no revelar los intereses que realmente estaban en juego, sino que se hizo todavía mayor cuando otros actores que conforman ese centro racional, desde el cual se toman las decisiones en materia de política exterior (think tanks, académicos, medios de comunicación) secundaron sin inmutarse el extremismo de Bush, dando legitimidad a su actuación internacional en los ámbitos local y mundial.

Así fue como diversas decisiones de política exterior, pero especialmente las concernientes a la guerra en Afganistán y la invasión de Irak, son las razones parciales que condujeron a Estados Unidos al caos económico y político que Obama heredó en 2008. Por un lado, el elevado gasto militar contribuyó al incremento del déficit presupuestal y de la deuda pública, por el otro, Estados Unidos se sumió en una crisis política interna y en el exterior producto de los factores que se enlistan a continuación: 
1) La práctica del unilateralismo, reflejado en la decisión de invadir Irak a pesar de la oposición de varios miembros del Consejo de Seguridad de la onu y la ausencia de pruebas fehacientes para ello (después se probó que las armas de destrucción masiva que el gobierno estadunidense dijo que Irak poseía no existían) (Blix, 2006), expuso críticamente la credibilidad y respetabilidad del liderazgo estadunidense en el mundo, al tiempo que otros actores, como China, aprovecharon los ocho años de la ausencia de una política exterior estadunidense equilibrada y efectiva -en términos de los temas a tratar y del ejercicio del poder- para perfilarse como potenciales actores decisivos en el concierto internacional. Los vacíos generados por Washington en el ejercicio del poder global fueron llenados hábilmente por algunos actores emergentes.

2) El ejercicio de la política exterior, predominantemente en manos del Departamento de Defensa, pasando por alto la opinión del Departamento de Estado. Se trató de una política exterior militarizada al extremo. Esto y la excesiva confianza en la tecnología militar condujeron al caos que hoy sigue dominando en Afganistán e Irak, con las implicaciones directas e indirectas en el resto de las respectivas subregiones.

3) Objetivos no cumplidos, pues al final de la administración Bush el mundo no era un lugar más seguro: ni el terrorismo ni los potenciales santuarios de los terroristas habían sido erradicados ni tampoco la red terrorista Al-Qaeda había sido derrotada ni mucho menos y tampoco su líder, Osama bin Laden, capturado. Y, hasta hoy, ni Afganistán ni Irak han alcanzado el estatus de democracias en el sentido más amplio de la palabra.

\section{ReCONDUCIR LA ESTRATEGIA: OBAma y EL PODER INTELIGENTE}

Ante esa crítica situación, era menester el replanteamiento de la estrategia de política exterior. El reto para el nuevo presidente era mayúsculo, en tanto que no solamente había que proteger a Estados Unidos de las amenazas tradicionales y mantener la condición de país dominante. También había que despejar y sanear el terreno que la administración Bush dejó minado en el ámbito esencial de sus alianzas tradicionales, con miras a recuperar el liderazgo estadunidense en el mundo, evitar un declive mayor del poder de Estados Unidos y, en ese sentido, emprender el camino hacia el cumplimiento de los compromisos esperados de Estados Unidos en política exterior.

La opinión pública (local e internacional) vivió un gran desencanto por el desempeño de la administración Bush, por lo que también demandaban un cambio en 
el comportamiento de Estados Unidos. ${ }^{3}$ Los aliados y diversos actores políticos internos de Estados Unidos pedían la retirada militar de Afganistán y de Irak, mientras que amplios sectores de la sociedad estadunidense demandaban una mayor atención a los asuntos de política interna, principalmente en los ámbitos de la economía y el creciente desempleo.

En este contexto, la elección de Barack Obama aumentó considerablemente las expectativas por tratarse de un político sui géneris: cosmopolita, intelectualmente bien dotado, profesionalmente educado en la misma Ivy League donde se formaron algunos de sus predecesores. Egresó de la carrera de Ciencias Políticas en la Universidad de Columbia y culminó la de Derecho en Harvard. Además, Obama pertenece a la minoría afroamericana, pero sin el pasado esclavista de sus pares (en este sentido, aunque miembro formal de esta minoría, también responde a la condición de mulato), con un discurso moderado, aunque propio de un político progresista que no responde al acartonamiento del establishment estadunidense. Por las características del candidato y su discurso conciliador y articulado, acompañados de una campaña presidencial muy bien estructurada, la opinión pública asumió que vivía un parteaguas histórico, en el marco del cual el comportamiento de este país podía cambiar y quizá volverse benevolente.

Obama cambió la estrategia de política exterior y decidió hacer uso del poder inteligente, definido como la combinación del poder duro de coerción y del suave de persuasión y atracción (Armitage y Nye, 2007; Nye, 2011), y que implica utilizar toda la gama del poder nacional, privilegiando el uso de la diplomacia y dejando en última instancia - pero sin descartarlo- el uso de la fuerza. El gobierno estadunidense reconoce que "para enfrentar los retos actuales, Estados Unidos [debe] utilizar [su] fuerza militar, diplomática, económica, de la información, legal y moral de forma equilibrada" (The White House, s.f.).

El término "poder inteligente" fue acuñado en 2004 por Suzanne Nossel, quien sugirió que tras la invasión a Irak, Estados Unidos debía renovar la doctrina del internacionalismo liberal, en la cual se estipula que

un sistema global de democracias liberales debería ser menos propenso a la guerra. Washington [...] debe ofrecer, por tanto, un liderazgo -diplomático, económico y no por ello menos militar- positivo para avanzar una amplia variedad de objetivos [...]. A diferencia de los conservadores, quienes se basan en el poder militar como la principal herramienta

\footnotetext{
${ }^{3}$ En una encuesta aplicada en veinticinco países en 2007 (ввС, 2007), el 49 por ciento de los encuestados percibía como negativa la influencia de Estados Unidos en el mundo; mientras que el 32 por ciento la consideraba positiva. Más del 50 por ciento desaprobaba la manera en que Bush abordaba ciertos temas: los programas nucleares de Irán y Corea del Norte, la guerra en Irak y el conflicto palestino-israelí.
} 
de la política, los internacionalistas liberales consideran igualmente importantes el comercio, la diplomacia, la ayuda extranjera y la expansión de los valores estadunidenses" (Nossel, 2004).

Partiendo de este enfoque, Estados Unidos reconoce en su nueva narrativa que no se encuentra solo en el mundo y que, por ende, para la resolución de diversos problemas regionales o globales se requiere de la intervención de diversos actores, llegando a la conclusión de que ni Washington puede resolver todos esos problemas por sí solo ni tampoco el resto del mundo puede resolverlos sin aquél (Obama, 2007).

No obstante, Estados Unidos no pierde de vista que el fin último es asegurar su statu quo en el mundo, por lo que a pesar de la emergencia de nuevos actores en la escena internacional, este país mantendrá el liderazgo. Para alcanzar su objetivo y mostrar una nueva cara al mundo -la de un Estados Unidos abierto al diálogo y a la cooperación con otros actores- hará uso de la diplomacia multilateral y mostrará interés por la defensa de los bienes públicos globales (Nye, 2002-2003), como la economía, el medio ambiente, los derechos humanos, entre otros. De este modo, Estados Unidos trataría de buscar el acercamiento con actores clave para la resolución de los problemas que también son vitales para sus objetivos e intereses -con los aliados tradicionales, como la Unión Europea y, particularmente, con los nuevos centros de influencia emergentes: China, India y Rusia, y con las naciones de creciente influencia, como Brasil, Sudáfrica e Indonesia (The White House, 2010a: 43-45).

En resumen, mediante el poder inteligente, Estados Unidos no intenta obligar a otros países a que hagan lo que Washington quiera (poder duro), sino que los otros hagan lo que éste quiere, porque ellos así lo desean y porque buscan y desean lo mismo (poder suave) (Nye, 2002-2003).

\section{LA AGENDA DE SEGURIDAD dE ObAMA}

En el marco de las crisis ya descritas, y desde el inicio de su mandato, Obama ha tenido que ser cauteloso al elegir qué temas de política exterior retomar y cómo hacerlo para avanzar en los intereses estadunidenses sin enfrentar riesgos innecesarios. ${ }^{4}$ Para ello decidió enfocarse en los temas que merecían atención urgente, toda vez que habían

\footnotetext{
${ }^{4}$ Esos intereses se definen como duraderos en la Estrategia de Seguridad Nacional de la administración de Obama y son 1) la seguridad de Estados Unidos, sus ciudadanos, sus aliados y socios; 2) una economía estadunidense fuerte y sostenible, dentro de un sistema económico internacional abierto y distendido; 3 ) respeto a los valores universales dentro y fuera de Estados Unidos; 4) un orden internacional guiado por Estados Unidos, que promueva la paz, la seguridad y la oportunidad mediante la cooperación (The White House, 2010a).
} 
puesto en riesgo la credibilidad y respetabilidad de este país en el mundo. De este modo, en la Estrategia de Seguridad Nacional de Obama, se citan entre los temas principales: el terrorismo, las armas nucleares, las guerras en Irak y Afganistán, las relaciones con el mundo musulmán, Irán, Corea del Norte, Rusia y China (The White House, 2010a).

\section{EL TERRORISMO Y LAS ARMAS NUCLEARES}

Obama no prosiguió con la lógica de la guerra global contra el terrorismo y los Estados que lo financian, aunque esto no significó que el tema haya sido desplazado de la agenda de seguridad. En la Estrategia de Seguridad Nacional queda asentado que ya no se trataría de una guerra global contra el terrorismo, sino que ahora Estados Unidos estaría en guerra contra Al-Qaeda y sus afiliados que intentaran atacar a Estados Unidos, sus aliados y socios. Asimismo, se hizo hincapié en evitar que los que son y que posiblemente se conviertan en Estados fallidos se vuelvan paraísos de terroristas. De este modo, Obama trató de reducir el uso, al menos desde el discurso, del término "guerra global contra el terrorismo", como lo hiciera Bush. Incluso en la misma Estrategia... resalta que "el terrorismo es una de muchas amenazas relevantes en la era global [pero que] el peligro más grave para el pueblo estadunidense y la seguridad global sigue proviniendo de las armas de destrucción masiva, particularmente las armas nucleares" (The White House, 2010a).

Precisamente, ante la preocupación del destino final de las armas nucleares, surgió la iniciativa estadunidense de reformar su estrategia nuclear mediante la cual se tomó la decisión de que las armas nucleares dejarían de ser uno de los componentes vitales de su política de seguridad. Este replanteamiento de la estrategia nuclear permitió a Estados Unidos, en primera instancia, retomar la relación con Rusia y firmar el tratado START II y, posteriormente, exhortar a otros países del globo, en el marco de la Cumbre sobre Seguridad Nuclear de 2010, a pronunciarse por un mundo en el que la producción y flujo de este tipo de armamento fuera controlado y en algunos casos reducido, a fin de evitar que cayeran en manos terroristas. Este conjunto de hechos ayudó a mostrar un rostro diferente de Estados Unidos en el mundo; no obstante, el objetivo era aplicar una serie de políticas que coadyuvaran a delinear una postura contra los programas nucleares de Irán y de Corea del Norte. Por ejemplo, se obtuvo el voto ruso en el Consejo de Seguridad de la ONU para reforzar las sanciones económicas contra Irán ante su renuencia a abandonar el programa nuclear.

A pesar de que acciones como la reforma de la postura nuclear se llevaron a cabo para, gradualmente, dar un viraje en política exterior y de que el enfoque en la guerra contra Al-Qaeda, y no la guerra global contra el terrorismo, hayan dominado la lógica 
del discurso de Obama, la guerra global ha tenido un lugar importante en sus políticas. Además, el uso de la fuerza militar para combatirlo -aunque al mismo tiempo ha sido conjugada con otros recursos del poder inteligente, como ha ocurrido en Pakistán- no ha sido reducido, sino al contrario, ha permanecido casi igual y en ocasiones ha superado al de la administración de George W. Bush. Por ejemplo, entre 2009 y 2012, Obama efectuó alrededor de 239 ataques encubiertos con drones (aviones no tripulados), cinco veces más de los 44 que la administración Bush aprobó (Rohde, 2012).

Pero la explicación a este comportamiento no radica en que Obama sea un belicista, sino que lo hecho forma parte del legado de Bush y del empoderamiento que en su momento adquirió el establishment militar y de inteligencia estadunidense, con las implicaciones negativas que tuvo en la legitimidad y respetabilidad global de Estados Unidos.

En primer lugar, Obama apostó por el uso de drones, en tanto que así se evitaba desplegar elementos castrenses en el terreno y, por tanto, ni se corre el riesgo de causar bajas militares que tanto inconforman a la población estadunidense ni de exacerbar los ánimos entre las poblaciones musulmanas que acusarían una ocupación del Tío Sam. ${ }^{5}$

En segundo lugar, se halla la legislación vigente, suscrita por la administración Bush en el marco de la guerra global contra el terrorismo. La Ley de Autorización del Uso de la Fuerza Militar es el sustento legal bajo el cual se estableció el uso de drones y la creación de la prisión de Guantánamo, la cual Obama prometió cerrar en campaña, promesa que incumplió y de la que se desistió casi al año de asumir la presidencia, en tanto que no hubo consenso interno para modificar esa ley. Como esa legislación, aún siguen vigentes otras más, mediante las cuales se empoderó a la CIA, encargada de hacer prevalecer una buena parte de las líneas de operación creadas desde la administración de Bush. ${ }^{6}$

De lo anterior se infiere que la estrategia de Obama en contra de Al-Qaeda y otros grupos terroristas tenga continuidad respecto de la guerra global contra el terrorismo de Bush, aunque en el discurso oficial se hable de una ruptura o cese de la estrategia de su predecesor. Al respecto, el Reporte de la Revisión Cuadrienal del Departamento de Defensa señala que Estados Unidos, sus aliados y socios "permanecen comprometidos en una guerra ampliada -política, militar y moral- contra Al-Qaeda y sus aliados en todo el mundo" (Department of Defense, 2010). Este hecho irremediable nos habla del grado en que, desde el interior del sistema que encabeza como jefe de Estado, se frenaría cualquier oposición a que se mantengan zonas de

\footnotetext{
${ }^{5}$ No obstante, ha habido severas críticas provenientes de los diferentes sectores del espectro ideológico (Commentary Magazine, 2013; Foreign Affairs, 2013; Cole, 2013).

${ }^{6}$ Sobre el papel de la CiA en este asunto, véase Mann, 2012: cap. 8, "CIA and the 'Aw, Shit!' Campaign".
} 
mando que siguen dominadas por la óptica clásica del régimen de seguridad nacional, cuyos remanentes persisten en la política internacional de Washington.

\section{IRAK Y AFGANISTÁN}

La atención prestada durante la administración de Obama a los dos frentes de guerra que tenía abiertos ha sido distinta para cada caso: por un lado, Afganistán se convirtió en la prioridad de su mandato, mientras que no fue así en el caso del conflicto en Irak. Esto se explica por lo siguiente: 1) la invasión a Irak, perpetrada entre marzo y mayo de $2003,{ }^{7}$ se convirtió en una guerra que carecía de lo necesario para ser catalogada como tal, además de que empezó a causar muchas bajas, razón por la cual el descontento entre la opinión pública aumentó, y 2) porque la administración de Bush había firmado un acuerdo en noviembre de 2008, en el que se estableció el 31 de diciembre de 2011 como fecha límite para el retiro de las tropas. Si bien Irak sigue viviendo en medio del caos y la inseguridad, por lo cual el primer ministro, Nuri al Maliki, intentó renegociar la estancia de las tropas estadunidenses con Obama, días antes de que se cumpliera la fecha límite, estos argumentos han guiado hasta ahora con relativo éxito la política de Obama en aquel país.

En contraste, la guerra en Afganistán se convirtió en una de las prioridades de la política exterior de la administración de Obama, esto debido a que para el gobierno mismo la situación en Afganistán era peor que en Irak, de tal modo que la permanencia de un frente abierto permitiría concentrar en ese país todos los esfuerzos ya emprendidos (The White House, 2009b). Además, se reconocía que los objetivos de la guerra no se cumplieron y también que el problema no sólo era Afganistán, sino también Pakistán, ya que este último era el santuario de los talibán.

Inicialmente, Obama llevó a cabo la evaluación de la estrategia que se había seguido durante la administración de Bush, sólo para percatarse de que en Afganistán la situación era realmente caótica, pues se carecía de objetivos claros, así como de una estrategia de salida (Woodward, 2010:48). De este modo, Obama planteó la reconducción de la estrategia en ambos frentes de guerra toda vez que la administración Bush había subestimado las condiciones -políticas, económicas y sociales- de ambos países y, por otro lado, había sobreestimado la efectividad de la tecnología militar confiando en que el uso de la fuerza sería uno de los elementos cruciales para producir

\footnotetext{
${ }^{7}$ El 20 de marzo de 2003 se invadió Irak y, tras cuarenta días, el 1º de mayo, Bush apareció ante los medios de comunicación para proclamar la victoria estadunidense en Irak, anunciando el fin de las principales operaciones de combate; no obstante, la guerra siguió su curso, sobre todo en respuesta a la insurgencia iraquí. El fin de las operaciones de combate lo anunció Barack Obama el 31 de agosto de 2010.
} 
las condiciones necesarias con el fin de implantar regímenes democráticos (Woodward, 2000; 2004).

Al anunciar el fin de la misión de combate en Irak el 31 de agosto de 2010, se ratificó el retiro de noventa mil efectivos de las tropas y la permanencia de cincuenta mil efectivos para llevar a cabo tareas de entrenamiento y apoyo a la policía y las fuerzas armadas iraquíes hasta su retiro, en diciembre de 2011 (The White House, 2010).

Para Afganistán y Pakistán se anunció una nueva estrategia que combinaba contrainsurgencia y contraterrorismo, por lo que Obama ordenó: 1) en Afganistán, el aumento a diecisiete mil tropas, y posteriormente a treinta y tres mil, sumando con ello aproximadamente cien mil elementos en el terreno (Belasco, 2011) y 2) el otorgamiento de ayuda económica a Pakistán para fortalecer su economía, con el fin de mermar la influencia de los talibán y de diversas redes terroristas que intentan cooptar a la población pakistaní y afgana. ${ }^{8}$

Esta estrategia, que permitió generar más y mejor información de inteligencia, y por la cual Washington no comunicó todos sus actos a los servicios de inteligencia pakistaníes (ISI), dio como resultado la operación llevada a cabo por la unidad más profesionalizada de los Navy Seals (marines estadunidenses), el Seal Team 6, y a través de la cual se capturó y ultimó a Bin Laden en su residencia de Abotabad, Pakistán el $1^{\circ}$ de mayo de 2011. Este hecho alentó aún más la rápida salida de las tropas de Afganistán, aun cuando ése haya sido uno de los objetivos de la guerra; ello debido a que, por un lado, la opinión pública consideró favorable el desempeño de Obama ante este acto, al tiempo que ya no continuaba apoyando la guerra ${ }^{9} \mathrm{y}$, por el otro, al hecho de que Washington asume que Al-Qaeda tal cual se le había conocido inicialmente, estaba a punto de disolverse (Miller, 2011). Así las cosas, en junio de 2011, Obama anunció el repliegue gradual de 33000 efectivos (10 000 para fines de 2011 y 23000 en 2012), manteniendo aún entre 66000 y 68000 tropas en el terreno (Katzman, 2011) que serán retiradas por completo en 2014.

Estos repliegues del frente de guerra permiten un margen mayor de maniobra para la política exterior estadunidense, no sólo en Medio Oriente y Asia Central, sino

\footnotetext{
${ }^{8}$ Desde el primer periodo de gobierno de Obama, se estimó invertir 7500000000 de dólares en ayuda para Pakistán, la que se ha ido dando por partes en el marco de programas y proyectos diversos de seguridad, crecimiento y desarrollo económicos; no obstante, ha habido ocasiones en las que el apoyo ha tenido que suspenderse como una forma de presionar al gobierno pakistaní a que se comprometa con una lucha frontal real contra terroristas. En otras ocasiones, la causa de la suspensión ha radicado en la expulsión de fuerzas estadunidenses de territorio pakistaní. De acuerdo con los Departamentos de Estado y Defensa y la agencia USAID, de 2009 a 2012 se invirtieron 4350000000 millones de dólares en ayuda para seguridad y poco más de 5300000000 de dólares en ayuda económica.

${ }^{9}$ En 2009, el 48 por ciento de los estadunidenses no apoyaba la guerra en Afganistán; mientras que el 45 por ciento sí era partidario (Boniface, 2009). Y aun cuando el resto de los objetivos de la guerra no se cumplieron, la popularidad de Obama subió 6 puntos (del 46 al 52 por ciento) tras la muerte de Bin Laden (Jones, 2011).
} 
también en otras regiones del mundo, principalmente Asia-Pacífico, que se anunció como una de las prioridades de la política exterior estadunidense. En cuanto a Medio Oriente, Estados Unidos no se mantiene alejado de la zona debido a que constituye un factor categórico de balance de poder regional. Además, el momentum que generó la primavera árabe, particularmente la crisis en Siria, hacen que su atención y presencia se mantengan.

\section{ACERCAMIENTO AL MUNDO MUSULMÁN}

El acercamiento con el mundo musulmán era, sin duda, uno de los asuntos más urgentes por tratar, toda vez que las guerras en Irak y Afganistán se percibieron como una ofensiva antimusulmana y desencadenaron un profundo sentimiento antiestadunidense no sólo en Medio Oriente, sino en el resto del mundo. Además, del relajamiento de las tensiones entre Estados Unidos y varios países del bloque islámico depende la reconducción de la estrategia de la política exterior estadunidense hacia esta región: parte central de la estrategia en tanto que un equilibrio gradual de poderes que coadyuvará parcialmente al mantenimiento de un papel predominantemente estadunidense en esa latitud.

El primer paso de Obama fue plantear -en el discurso que pronunció en la Universidad de El Cairo, en junio de 2009- un recomienzo de las relaciones. Ahí, el mandatario estadunidense refrendó su compromiso con el desarrollo y bienestar de la región, enfocándose principalmente en los temas económicos y de promoción de la democracia y los derechos humanos (The White House, 2009a). El discurso causó los efectos deseados, pues éste, en combinación con la elección de Obama en 2008, ya habían generado grandes expectativas entre la población árabe (Arab American Institute Foundation, 2011).

La segunda oportunidad para fortalecer el compromiso con la región, y que ha permitido observar mejor el despliegue de la estrategia de poder inteligente, se suscitó a raíz de la primavera árabe. Obama recibió con beneplácito la decisión de la población de los países donde se registraron (y siguen registrándose) protestas que demandan la caída de los regímenes autoritarios. A la vez, aunque no en forma de un involucramiento directo, se comprometió a apoyar la transición hacia la democracia; y en el caso de Egipto y Túnez, en donde fueron derrocados los regímenes de Hosni Mubarak y de Zine El Abidine Ben Ali, respectivamente, aplicó una política para la reactivación de las economías de ambos países, lo cual expresó en un discurso en mayo de 2011 en Washington, al que, por su contenido y continuidad con lo expresado en el de El Cairo, se le ha denomina "El Cairo 2" (The White House, 2011). 
Por su parte, la cautela con que Obama atendió el asunto de Libia, al no intervenir directamente y optar por la vía del multilateralismo recurriendo al Consejo de Seguridad de la ONU para la resolución del problema, conjugó los elementos del poder inteligente, lo cual se percibió como la reiteración de la continuidad de la estrategia de imponer una nueva imagen de Estados Unidos en el mundo.

No obstante, los efectos favorables para la política inteligente del gobierno de Obama en esta región se han desvanecido por diversas razones: en primer lugar, la oposición estadunidense a la petición de la autoridad palestina de ser miembro de la ONU provocaría que los esfuerzos previos para acercarse al mundo musulmán se vieran minados. Esta resistencia se advierte en la región como un acto de hipocresía, pues se apoya la autodeterminación de los países que han desencadenado la primavera árabe y, al mismo tiempo, se niega la independencia palestina; ${ }^{10}$ no obstante, es posible que Estados Unidos no cambie su postura ante este diferendo, ya que Israel es un aliado clave (e ineludible) en la región, de ahí que continúe pronunciándose para que el acuerdo en este tema sea producto de las negociaciones entre estos países, no a través de una decisión tomada en el seno del Consejo de Seguridad de la ONU.

En segundo lugar, el equilibrio de poder en la región corre el riesgo de alterarse a causa de la crisis en Siria, debido a la renuencia de Bashar al Assad de dimitir de su cargo como presidente, así como por la fuerza y las armas químicas que ha utilizado en contra de la población siria, lo que -hasta el momento en que se escribe este artículo- ha cobrado la vida de más de cien mil personas. Y, lo que es peor, esta situación ha puesto al borde del precipicio la estrategia de poder inteligente de la administración de Obama, contra la cual han arreciado las críticas de la opinión pública local e internacional.

Por un lado, hay quienes señalan que su actuación no ha estado a la altura del líder del país que se erige como principal potencia mundial vis-à-vis quienes critican que el Premio Nobel de la Paz no se puede permitir una intervención militar que reviviría un escenario iraquí, suceso que pervive en la memoria colectiva como traumático.

A pesar de la crisis humanitaria en ese país y de que se vivieron días tensos cuando Obama manifestó su decisión de intervenir militarmente en Siria, en el fondo Barack Obama se inclinaba por la resolución del conflicto a través de la diplomacia. Aparentemente la clave para evitar la intervención militar -no necesariamente para detener la masacre y la guerra civil- parece apenas dibujarse a raíz del acuerdo alcanzado por Estados Unidos y Rusia (socio principal de Siria), en el cual se estableció no intervenir a cambio de que el presidente sirio entregara todas sus armas químicas (Putin, 2013).

\footnotetext{
${ }^{10}$ En este sentido, la opinión favorable hacia Obama en 2009, en 2010 disminuyó según el reporte “Arab
} Attitudes 2011" (Arab American Institute Foundation, 2011). 


\section{"El siglo del Pacífico estadunidense"}

La política exterior del gobierno de Bush descuidó varios temas y regiones del mundo al concentrarse sólo en la lucha contra el terrorismo. Si el objetivo de Estados Unidos era mantener su papel como actor predominante, la mejor manera de hacerlo -al menos desde la perspectiva del poder inteligente- no era por la vía de la coerción, sino la actuación en conjunto con el resto de los actores, para así ejercer un mayor poder suave y evitar que otros se perfilaran como potenciales competidores por la supremacía mundial.

En este camino y tenor se halla China, cuyo crecimiento (principalmente económico) ha sido rápido en un periodo relativamente corto, en comparación con el de Estados Unidos, superando a Japón como la segunda economía más grande del mundo; ${ }^{11}$ no obstante, Obama ha dejado claro que a China se la ve como competidora, incluso como una amenaza para la influencia estadunidense no sólo en la región Asia-Pacífico, sino también en el resto del mundo. Al respecto, basta recordar cuando, en el marco del discurso del Estado de la Unión de 2011, el presidente señaló que la generación estadunidense actual enfrenta su "momento Sputnik" y requiere, por tanto, de la inversión del Estado en investigación, educación e infraestructura que países como China sí se habían preocupado por realizar y que los coloca en una posición de relativa ventaja en el concierto internacional.

Ciertamente esta idea surgió en el contexto de la crisis económica estadunidense, lo cual obliga a buscar opciones para reactivar dicha economía, pero también fue un llamado a afrontar al ascenso de China como potencia económica. El mensaje es claro: Estados Unidos intenta no perder su poderío a manos de los chinos, lo cual, a la larga, devendría en el aumento de su poderío militar y político, cuyo resultado será la presencia de un nuevo actor decisorio en el orden internacional y, con ello, un inconveniente reajuste del orden mundial, alejado de la tradición liberal que le dio origen.

Para evitar tal escenario, sobre lo que además existe el consenso de los aliados de Washington, Estados Unidos ha esbozado políticas que imitan una política de contención, la cual apoyan varios actores de la región, ya que tampoco ellos aceptan la creciente influencia china y, por ende, se mantienen cómodos con la presencia y protección de Estados Unidos en la región. Entre dichos aliados se encuentran Japón, los miembros de la Asociación de Naciones del Sureste Asiático (algunas de las cuales han entrado en confrontación con China por la disputas de las islas del Mar del Sur y del Este de China, todo lo cual implicaría que China adquiriera derechos sobre el petróleo y el gas ahí existente); incluso India, que aunque no forma parte de aquella

\footnotetext{
${ }^{11}$ Entre 1999 y 2009, el porcentaje del PIB mundial de Estados Unidos cayó del 23 al 20 por ciento, mientras que el de China ascendió del 7 al 13 por ciento.
} 
región, está atenta al posible repunte de China en tanto que es aliada de Pakistán, su rival histórico.

En este contexto, Estados Unidos, como miembro del Acuerdo de Asociación Transpacífico, ${ }^{12}$ ha pedido que este tratado comercial se convierta en un pacto comercial del siglo xxI; es decir, que permita incluir o desarrollar nuevos sectores y tecnologías, lo cual orbita en torno a las necesidades estadunidenses de contar con un mercado para sus exportaciones que ayude a la recuperación económica y a la creación de nuevos empleos (Office of the US Trade Representative, 2011) toda vez que la región Asia Pacífico representa aproximadamente el 60 por ciento del PIB mundial y aproximadamente el 50 por ciento del comercio mundial (Fergusson y Vaughn, 2009). Asimismo, Estados Unidos busca la ampliación del Acuerdo mediante la adhesión de nuevos miembros, como Japón, Corea del Sur, Indonesia y Filipinas.

Más aún, la región Asia-Pacífico se convertirá en una de las prioridades de política exterior de Estados Unidos. Así lo señaló la exsecretaria de Estado Hillary Clinton, al decir que "el futuro de la política se decidirá en Asia, no en Afganistán ni en Irak y Estados Unidos estará en el centro de la acción”, razón por la cual, durante la próxima década, Estados Unidos tendrá la tarea de invertir esfuerzos "diplomáticos, económicos, estratégicos y de otro tipo" en dicha región (Clinton, 2011); de tal manera que "el siglo xxi será el siglo del Pacífico estadunidense" (Department of State, 2011).

Por otro lado, uno de los pasos que ha dado el gobierno estadunidense por afianzar su presencia en la región fue el anuncio, el 16 de noviembre de 2011, del despliegue de una base militar en la ciudad de Darwin, al norte de Australia (lo cual no ha agradado a Pekín), en un acto que Estados Unidos ha calificado como de protección al comercio de la región, pero que en realidad responde a la cautela ante el creciente poderío económico y militar chino. En esa misma tesitura se ubicó el anuncio del exsecretario de Defensa de Estados Unidos, Leon Panetta, acerca de que en el 2020 el 60 por ciento de la flota estadunidense se encontrará en el Pacífico. ${ }^{13}$

\section{CRíticas RePublicanas O DE LA CEGUERA DE LOS PATRIOTAS EN SILLA DE RUEDAS}

Cada uno de los movimientos de Obama, tanto en política interna como exterior, ha sido sometido a un estricto escrutinio, aspecto en el que la oposición republicana

\footnotetext{
12 Tratado del que también son miembros Australia, Brunei, Chile, Nueva Zelanda, Malasia, Perú, Singapur y Vietnam.

${ }^{13}$ Este anuncio lo hizo Panetta en un discurso que pronunció en el International Institute for Strategic Studies (Shangri-La Dialogue), en Singapur, el 1º de junio de 2013.
} 
ha invertido una energía excepcional. Los resultados de la elección presidencial de 2008 (el 53 por ciento de Obama frente al 46 por ciento de McCain) retrataron una sociedad estadunidense polarizada, particularmente ante el hecho de que un afroamericano ocupara la presidencia de la nación que históricamente ha dado importancia a la jerarquía de raza. ${ }^{14}$

Si bien el "pensamiento racial" predomina entre diversos sectores de la colectividad estadunidense, los sectores ultraconservadores de la elite política estadunidense, encabezados hoy por el movimiento del Tea Party, se han encargado de expandirla aún más y de reforzar el concepto de que Obama no es el hombre idóneo para gobernar el país. Estos sectores no perdonan a Obama que sea presidente, porque ellos consideran que es un outsider, un joven político progresista y, desde luego, una "anomalía" WASP (white, anglosaxon and protestant people). Ante esto han recurrido a una estratagema exitosa en la historia social estadunidense y lo han acusado de ser socialista, musulmán y no estadunidense, todo ello con la pretensión de deslegitimar sus acciones; no obstante, lejos de "lacerar" la presidencia de Obama, han conseguido poner en jaque al sistema político estadunidense y producir una crisis dentro del Partido Republicano (PR) de consecuencias inciertas.

Las críticas a Obama desde los segmentos extremistas de la política (generalmente sin sustancia) han estado principalmente orientadas no tanto al manejo de la política exterior, como al manejo de la interna, pues ésta es la que interesa en forma central al votante común. ${ }^{15}$ En este ejercicio, los republicanos han acusado confusión y demagogia que bien pueden deberse al hecho de que no cuenten con propuestas ni voceros que articulen una idea siquiera sensata sobre cómo recuperar el liderazgo internacional, y en esto han quedado vacíos y en el vacío. Así, el tema de la crisis económica ha quedado atrapado en medio del desacuerdo político originado por la lucha de los ultras por dañar a Obama.

Por ejemplo, en agosto de 2011, se llegó al grado de hablar sobre la posible suspensión de pagos, ante la ausencia de un acuerdo temprano en el Congreso que permitiera elevar el techo de la deuda. Tal acuerdo se alcanzó, pero tarde (en la fecha límite) y, encima de todo, semiacabado, ya que varios puntos del mismo quedaron sujetos a renegociación posterior, lo que puso de nuevo en el cuadrilátero político a modera-

\footnotetext{
${ }^{14}$ Michael Hunt señala tres elementos característicos de la política exterior estadunidense a lo largo de la historia, los cuales -sostiene- que han significado fundamentos ideológicos y de política pública desde la fundación de Estados Unidos: 1) visión de grandeza nacional, 2) jerarquía de raza, 3) límites impuestos a las revoluciones (Hunt, 1987: 17-18).

${ }^{15}$ Como lo muestra una encuesta publicada por Gallup pocos días antes de las pasadas elecciones de noviembre, en la que el 37 por ciento de los estadunidenses encuestados dice que la economía en general es el problema más serio que enfrenta su país, seguido por el desempleo y el déficit fiscal. Mientras que ningún tema relacionado con la política exterior es mencionado siquiera por un 5 por ciento de la población.
} 
dos y ultras y, peor aún, en diciembre de 2012 estuvo a punto de conducir al precipicio fiscal y en marzo de 2013 llevó a lo que se denominó "secuestro del presupuesto". La historia se repitió a finales de septiembre de 2013, generando una crisis más con los orígenes de intransigencia señalados.

Este acuerdo impuso varios recortes presupuestales, uno de los cuales recaerá a lo largo de la próxima década en el sector defensa, con quinientos mil millones, lo cual sugiere que Obama tendrá que ser cuidadoso al definir sus prioridades en el exterior. Esta reducción ha suscitado descontentos entre los sectores conservadores, pues temen que ante la limitación de la política estadunidense en el exterior se suscite un rápido declive de Estados Unidos en el mundo; ${ }^{16}$ no obstante, Parent y MacDonald (2011) advierten que estos señalamientos son infundados, toda vez que la historia, no sólo de Estados Unidos, sino de otras grandes potencias, ha mostrado que no es precisamente la reducción de la preponderancia militar en el mundo la que ha conducido a la pérdida de supremacía internacional en términos generales. En algunos casos, ha ocurrido lo contrario, ya que grandes gastos militares han llevado a quiebras económicas (como ocurrió con la URSS), mientras que importantes y excesivos compromisos en el exterior han causado que no todos ellos sean cumplidos - para muestra, basta ver a Irak y Afganistán.

No obstante, en el marco de las primarias republicanas, las posturas en materia de política exterior de los potenciales candidatos sugirieron el retorno al poder duro de Bush. Por ejemplo, los ocho contendientes (Jon Huntsman, Mitt Romney, Rick Santorum, Newt Gingrich, Ron Paul, Herman Cain, Michele Bachmann, Rick Perry -la mayoría sujetos a la presión del Tea Party-), concordaban en que, de convertirse en presidentes, la política hacia Pakistán, Siria e Irán sería agresiva y se usaría incluso la fuerza militar en estos últimos; continuarían con el respaldo al Israel del caprichoso Benjamín Netanyahu y se perseguiría con dureza que China abandonase las prácticas desleales en el ámbito comercial. Algunos no dudaron en retomar la política del despliegue de un escudo antimisiles en Europa del Este, y seis de ellos estaban decididos a utilizar el ahogamiento simulado (waterboarding) ${ }^{17}$ entre los prisioneros de guerra (quienes se oponían eran Paul y Huntsman).

Semejante fue la línea que siguió Mitt Romney, nominado por el PR para contender por la presidencia. Durante ese tiempo, él y su equipo de campaña se dedicaron

\footnotetext{
${ }^{16}$ Paradójicamente para los ultras republicanos, "los absurdos recortes", como los llamó Obama, ya causan estragos en el poder de despliegue militar y en los controles fronterizos con México, que han generado una disminución de la vigilancia fronteriza por parte de la Patrulla Fronteriza.

${ }^{17}$ Es una técnica de interrogación catalogada como tortura. El tema ha dado mucho de qué hablar luego de la nominación de John Brennan para dirigir la CIA, pues, quien colaborara en esa misma dependencia durante la administración de Bush, dijo que se había opuesto rotundamente a tales técnicas; sin embargo, ante el Comité de Inteligencia del Senado, en su audiencia de confirmación, reconoció que no intentó detener dicho programa.
} 
a descalificar los logros de Obama en política exterior y a intentar capitalizar algunos descuidos de su administración, como el ataque al Consulado estadunidense en Bengasi, Libia, donde murió el embajador Christopher Stevens. Al final, las posturas republicanas que sugerían retomar el poder duro como primer recurso pusieron de manifiesto su negligencia en temas de política exterior, sugiriendo que dentro del PR varios no han tomado en cuenta los efectos nocivos de la política exterior de la era Bush, por lo que siguen sin ser conscientes de que es mejor ejercer un buen liderazgo, en vez de imponer dogmáticamente la hegemonía estadunidense, tal y como en su momento sugirió Brzezinski (2004). ${ }^{18}$ A estas alturas del siglo, el Partido Republicano parece acercarse mucho a la grotesca caracterización que de éste hiciera Gore Vidal, cuando dijo que el PR no es un partido, sino un estado de ánimo, como la juventud hitleriana, basada en el odio.

\section{CONCLUSIONES: ALCANCES Y LÍMITES}

\section{DE LA POLÍTICA EXTERIOR ESTADUNIDENSE}

La preocupación por el quehacer de la política exterior estadunidense, tanto en el primero y segundo mandatos de Obama, surge en medio de 1) la pérdida histórica del liderazgo relativo de Estados Unidos en el mundo, 2) el desacuerdo político y la crisis presupuestal y 3) el contexto pre y poselectoral de 2012, en el que prevaleció y prevalece la polarización. Por estas razones, es aún más importante que la política exterior estadunidense evite excesos, es decir, no comprometerse con más asuntos de los que puede ser capaz de afrontar $y$, por ende, no invertir innecesaria e inflexiblemente grandes cantidades de recursos económicos en actividades en el exterior. Inicialmente, la estrategia de poder inteligente estuvo encaminada, sobre todo, a lidiar con el primer punto, pero poco a poco ha tenido que adaptarse a las necesidades que demandan el segundo y tercer puntos.

En primer lugar, en lo que se refiere a la recuperación del liderazgo estadunidense, la del poder inteligente se ha percibido como una estrategia que ha mostrado más ventajas que desventajas para Washington. Por ejemplo, se ha evitado, en el marco de un clima más amable, la confrontación directa con países con los que la relación bilateral ha estado en las tres últimas décadas plagada de desencuentros (como

\footnotetext{
${ }^{18}$ La nominación de Charles Hagel como nuevo secretario de Estado es una muestra clara de que el poder inteligente seguirá siendo el medio para alcanzar los objetivos en el exterior planteados por Obama. Hagel es un republicano y veterano de la guerra de Vietnam; sin embargo, ha sido crítico de la invasión a Irak de 2003, del lobby judío y de las grandes dimensiones que ha alcanzado el Pentágono en la política de seguridad y defensa estadunidenses.
} 
Rusia, Irán, Corea del Norte y Venezuela); también se han generado las condiciones para que las decisiones de carácter internacional se tomen por vía de la diplomacia multilateral (por ejemplo, la intervención de las fuerzas de la OTAN en Libia). Asimismo, el interés por el bienestar global (calentamiento global, derechos humanos, sostenibilidad, estabilidad económica y financiera) se ha puesto de manifiesto en la práctica y se ha mostrado disposición al diálogo (algunos gestos han sido más de efecto mediático) con los actores que habían sido desairados o con los que se había provocado un distanciamiento por el voluntarismo negligente de Estados Unidos (por ejemplo, con la Unión Europea, el mundo musulmán, y países de las regiones Asia-Pacífico y de América Latina, entre ellos Cuba).

Se reconoce aquí que esta estrategia no ha logrado todos sus objetivos. Para muestra basta ver el diferendo palestino-israelí, así como la falta de diligencia en el alcance de los consensos básicos en temas de la agenda global, como la crisis económica y financiera y el medio ambiente; no obstante, se hace hincapié en que la estrategia de poder inteligente es, en principio, una forma relativamente ventajosa para avanzar en el logro de los intereses, objetivos y valores de Estados Unidos en el mundo, y no un medio para solucionar el conjunto de problemáticas que aquejan a la comunidad internacional en su totalidad. Aunque esporádicamente impactaría en la ampliación de la democratización multilateral del sistema mundial y el rediseño de su arquitectura, la estrategia de poder inteligente busca la creación de un entorno seguro para Estados Unidos, el cual hoy se ve relativamente constreñido por la pérdida de liderazgo y el ascenso de otros actores emergentes que quizá pudieran convertirse en coactores predominantes del concierto internacional. Por ello, en su segundo mandato, Obama mantiene la idea de encaminar la resolución de los problemas que habían derivado en la pérdida de liderazgo y en retomar las relaciones con los nuevos actores emergentes, con el firme empeño de resituarse como un actor predominante en lo que se refiere a la plausible redefinición de la arquitectura del sistema internacional. ${ }^{19}$

La estrategia de política exterior de este segundo mandato no dista, en forma ni en fondo, del primer periodo presidencial. De hecho, los primeros cuatro años de los demócratas en el poder, con Hillary Clinton como secretaria de Estado, estuvieron destinados a reconfigurar en el terreno la estrategia de política exterior para poner en orden el desastre que dejó la administración de Bush.

El segundo mandato parecía proponerse consolidar todo el capital político adquirido; sin embargo, surgieron algunos inconvenientes que de nuevo pusieron en duda

\footnotetext{
${ }^{19}$ No obstante, el gobierno de Obama ha resaltado la importancia del multilateralismo. La exsecretaria de Estado Hillary Clinton señaló, en enero de 2009, frente al Comité de Relaciones Exteriores del Senado: "Estados Unidos no puede resolver los problemas más urgentes por sí solo, y el mundo no puede resolverlos sin Estados Unidos".
} 
la credibilidad de Estados Unidos y la del propio Obama. Esto se suscitó a raíz de la información filtrada por un extécnico de la cia y contratista de la Agencia de Seguridad Nacional, Edward Snowden, a través de la cual se dio a conocer que Estados Unidos posee un programa informático de vigilancia PRISM (por sus siglas en inglés), con el cual no sólo se mantiene al tanto de los movimientos de los estadunidenses, sino que también sirvió para espiar a algunos de sus socios en el mundo. El problema se agravó cuando Snowden huyó a Rusia para pedir asilo y refugiarse de la policía estadunidense que lo acusaba de traidor a la nación. Invariablemente, la relación entre Putin y Obama se tensó, pues el mandatario ruso se negó a extraditar a Snowden y terminó por concederle el asilo.

Como ya se señaló, las relaciones entre ambos países se han complicado aún más como resultado del conflicto sirio. Rusia ha bloqueado, junto con China, las medidas que en el Consejo de Seguridad de la ONu se han propuesto para detener la masacre de la población siria -contra la que recientemente se han usado armas químicas- y suministrar los recursos necesarios para tal efecto. Recientemente, Obama y Putin llegaron a un acuerdo sobre el tema, empero, ha sido el presidente ruso quien lo ha capitalizado, incluso en forma delirante, al ostentarse como un nuevo líder moral frente a un conflicto en el que Moscú tiene las manos manchadas. Destacamos que Putin se benefició de la negativa del Parlamento británico y del Congreso estadunidense de apoyar cualquier medida unilateral. A esto se abona la resistencia de Alemania y, con excepción de Francia, la de la Unión Europa. A casi un año después de su reelección, Obama contabilizaría varios logros de su política de poder inteligente, pero cualquier movimiento erróneo en torno al asunto sirio - al menos por ahora o frente a cualquier otra situación similar en el futuro pondrá en peligro lo poco que avance en esta estrategia.

En segundo lugar, está la crisis presupuestal. Si bien surgen temores ante el hecho de que esta situación limitaría la política de seguridad y defensa estadunidenses, también existen argumentos que nos permiten sugerir que se obtendría ventaja de este aspecto. Limitar el uso del poder militar es acertado en un contexto en el que no hay aceptación (local e internacional) por su utilización, la cual quizá agrave los escenarios, ya de por sí caóticos (como ocurrió en Afganistán y en Irak) o bien, por llegar a excesos demostrados del uso injustificado del poder militar, como ocurrió en Irak. Además, la administración de Bush dejó una lección: el exacerbado uso de la fuerza no necesariamente conduce a obtener el título de poder supremo del mundo ni a la estabilidad regional, mucho menos a tener control sobre los equilibrios regionales. En realidad, es la manera en que se utilice, en combinación con los otros elementos de poder (económico y político) lo que coadyuvará a acercarse a tal posición. La transición que supone lograr la recuperación del liderazgo y legitimidad internacional 
estadunidense, iniciada por Obama desde su primer mandato, probablemente se consolide durante el segundo, de conservarse el equilibrio interno y externo necesarios para ello. Según lo apuntó en su último discurso sobre el Estado de la Unión, se proseguirá con la estrategia de poder inteligente, intentando mostrar la cara de un país cooperativo e interesado en el bien común, para posiblemente recuperar legitimidad y contar con la autoridad para ejercer una postura influyente y predominante en la toma de decisiones del actual mundo globalizado, sin que ello signifique necesariamente violentar en alguna forma al resto de los actores.

Conviene anotar que aun cuando en la administración de Obama persisten agudas divisiones políticas, éstas son más profundas en el Congreso estadunidense en cuanto a los temas de política interna que externa. Ello no significa que demócratas y republicanos tengan las mismas ideas respecto de la forma de perseguir los intereses en el exterior; no obstante, será más fácil conseguir el acuerdo en tanto que 1) no todos los temas de política exterior ponen en juego las preferencias partidistas del electorado estadunidense y 2) se trata de mantener de manera congruente la postura del país ante el mundo, el cual sigue una tendencia hacia la reconstrucción y consolidación de un entorno seguro para Estados Unidos. Y parece que todo dependerá del alcance para encontrar condiciones que permitan pensar que se ha conseguido cierta civilidad en el ámbito de la búsqueda de los consensos. Este escenario, sin embargo, se ve permanentemente oscurecido por la irracional intransigencia antiobamista que prevalece en el seno dominante del PR, así como dentro del Congreso, tesis sostenida en el curso de este artículo.

En tercer lugar, pero no menos importante, los resultados de la elección presidencial de 2012 muestran a un electorado más preocupado por lo que ocurra con la economía estadunidense y el gasto social que por la política exterior, lo que hizo que las propuestas de los candidatos en este tenor no fueran determinantes. Obama, desde luego, ha mostrado interés por el tema de la crisis económica, planteando reformas internas, incluso buscando oportunidades en el exterior, a través del Acuerdo Transpacífico y de la creación del Acuerdo Trasatlántico de Comercio e Inversión con la Unión Europea. Empero, los sectores ultraconservadores continúan a la cabeza de la oposición de las iniciativas del presidente, lo que ha imposibilitado fehacientemente la reactivación económica y ha provocado que el déficit, la deuda y el desempleo continúen elevados. Como muestra de la antipolítica de los republicanos, primero se tuvo la preocupación de caer en el "abismo fiscal" y, más recientemente, de las consecuencias de no haber evitado el secuestro presupuestal (sequester).

No obstante, en la reciente elección hubo un obstáculo para que los sectores conservadores obtuvieran los votos suficientes que les permitieran recuperar el Ejecutivo y obtener la mayoría en ambas cámaras: las divisiones internas. Si bien una 
gran parte de las críticas al desempeño de la administración de Obama proviene de la oposición republicana, no todos sus miembros son ultraconservadores y seguidores del radicalismo del Tea Party que, por cierto, parece actuar con negligencia ante la crítica situación económica del país (por ejemplo, al imponer la aprobación de un presupuesto con recortes absurdos o, bien, al no apoyar las iniciativas de Obama para mejorar la economía). Esas posturas han incrementado la opinión poco favorable de los estadunidenses hacia este movimiento. De paso, el Partido Republicano también resultó afectado en las elecciones, toda vez que mostró excesiva tolerancia, cuando no complicidad, con un movimiento claramente autoritario, intolerante y regresivo. La derrota electoral republicana en las presidenciales y el Senado ha sido un factor que ha orillado a sus miembros a moderar posturas. Así es como decidieron aceptar (con varias condiciones) negociar una reforma educativa y otra migratoria que, aunque se halle en serio peligro por las crisis internas y externas recientes, no queda claro si podría retomar el buen camino.

Estas circunstancias -la pérdida de liderazgo estadunidense en el mundo, la crisis económica, los recortes al gasto de defensa y la crisis política interna- representan un panorama de gobernabilidad complejo para la administración de Obama. Por lo demás, no obstante esto, aun cuando el panorama no sea claro, esto no significa que el poderío estadunidense se encuentre cerca de su fin como para ceder el paso a nuevos actores predominantes en el concierto internacional.

Su poderío económico se encuentra aunque debilitado, relativamente afectado, en tanto que su deuda ha rebasado su PIB y su crecimiento ha disminuido (el 2.9 por ciento en 2010 respecto de 2009), pero aún sigue siendo la primera economía mundial con un PIB de 14.5 billones de dólares, mientras que el de China es de 5.8 billones de dólares (The World Bank, s.f.) y se ubica, después de ésta, como el país manufacturero más grande del mundo. Su poderío y gasto militar siguen siendo superiores a los de otros países, ya que hasta el año 2010 se ha mantenido como el país que más recursos destina a esta esfera, con 698000 millones de dólares anuales, equivalentes al 4.8 por ciento del PIB estadunidense (Perlo-Freeman et al., 2011). ${ }^{20}$ En cuanto al gasto público, en 2009 destinó el 16.2 por ciento del PIB, tanto en salud como en educación (United Nations Development Programme, 2011) y en 2008, en el ramo de ciencia y tecnología, se invirtió el 2.82 por ciento (The World Bank, s.f.).

Este conjunto de indicadores sugieren que no se puede hablar de un declive estadunidense absoluto. ${ }^{21} \mathrm{Si}$ bien su crecimiento económico es bajo, en comparación

${ }^{20}$ En segundo lugar, se ubica China con aproximadamente 119000 millones de dólares, seguido de Gran Bretaña, con un gasto anual de 59.6000 millones de dólares, Francia con 59.3000 millones de dólares, y Rusia con aproximadamente 58.7000 millones de dólares.

${ }^{21}$ En 2007, el gasto porcentual del PIB en educación y ciencia y tecnología fue, en el caso de Brasil, del 5.2 y el 1.1 por ciento, respectivamente; en Rusia, del 7.4 y el 1.12 por ciento; en India, del 3.3 y el 0.8 por ciento, y 
con los BRICS, ${ }^{22}$ éstos no destinan gran parte de su gasto a sectores clave para un desarrollo más sostenible, incluso aún tienen un largo camino por recorrer en otros temas, como distribución del ingreso, pobreza, desigualdad y derechos humanos. Finalmente, en el aspecto político, Estados Unidos sigue prevaleciendo como el actor central en los grandes temas de la agenda internacional y, para algunos países, su intervención en algunos de esos temas es fundamental, toda vez que mantiene virtudes como un equilibrador de poder.

Ciertamente, el panorama es abrumador al contar con poco margen de maniobra para actuar, sobre todo en materia de política exterior. No obstante, la estrategia emprendida parece haber colocado a Estados Unidos frente a una oportunidad histórica para corregir los efectos de sus excesos en el mundo, a fin de ir recuperando los espacios que perdió en el entorno global. Además, la forma de lograrlo no dependerá únicamente de la gran cantidad de recursos y capacidades con que se cuente, sino de la optimización de ambos, de los mecanismos que utilice para alcanzar los objetivos, de la capacidad para desarrollar una estrategia coherente y constructiva, de la buena fortuna en lo que al surgimiento de crisis en el exterior se refiere y, en consecuencia, de un hábil liderazgo para aplicar medidas de contención; es decir, de contar con el conjunto de elementos necesarios para posibilitar el ejercicio exitoso de la estrategia de poder inteligente.

\section{FUENTES}

\section{Arab AMERICAN INSTITUTE Foundation}

2011 "Arab Attitudes 2011", en <http:/ / aai.3cdn.net/5d2b8344e3b3b7ef19_xkm6ba4r9.pdf $>$.

\section{Armitage, Richard y Joseph S. Nye, Jr.}

2007 CSIS Commision on Smart Power. A Smarter, More Secure America, Washington, D.C., Center for Strategic and Internationl Studies, en <http: / csis.org / files / media/csis/pubs/071106_csissmartpowerreport.pdf $>$.

en China, del 3.3 y el 1.44 por ciento, respectivamente. En 2009, el gasto porcentual del PIB en salud en Brasil fue del 9 por ciento; en Rusia, del 5.4 por ciento; en India, del 4.2 por ciento y en China del 4.6 por ciento (The World Bank, s.f.; OCDE, s.f.).

${ }^{22}$ El crecimiento del PIB estadunidense de 2009 a 2010 fue de 2.9 por ciento; mientras que el de Brasil fue del 7.5 por ciento, el de Rusia, el 4 por ciento; India, el 9.7 por ciento y China, el 10.3 por ciento (The World Bank, s.f.). 
$\mathrm{BBC}$

2007 “World View of US Role Goes From Bad to Worse", en <http: / news.bbc.co.uk/ 2/shared/bsp/hi/pdfs/23_01_07_us_poll.pdf $>$, enero.

BELASCO, AMY

2011 "The Cost of Iraq, Afghanistan, and Other Global War on Terror Operations Since 9/11", Congressional Research Service, en <http: / / www.fas.org/sgp/ crs / natsec/ RL33110.pdf>, consultada el 29 de marzo.

BLIx, Hans

2006 La reforma de las Naciones Unidas y las perspectivas futuras para el desarme, México, CISAN, UNAM, Cuadernos de América del Norte, 7.

\section{BONIFACE, PASCAL}

2009 "Afghanistan: le pari d'Obama", Affaires-stratégiques.info, Institute de Relations Internationales et Stratégiques, en <http: / / www.affaires-strategiques. info/spip.php?article2456>, 3 de diciembre.

BRZEZINSKI, ZBIGNIEW

2004 The Choice. Global Domination or Global Leadership, Nueva York, Basic Books.

BUZAN, BARRY

1992 "New Patterns of Global Security in the Twenty-First Century", International Affairs, vol. 67, no. 3 .

BYMAN, DANIEL

2013 "Why Drones Work", Foreign Affairs, vol. 92, no. 4, julio-agosto.

Clinton, Hillary

2011 "America's Pacific Century", Foreign Policy, en <http:/ / www.foreignpolicy. com/articles/2011/10/11/americas_pacific_century>, noviembre.

Cole, David

2013 “What's Wrong with Obama's Drone Policy?", The Nation, 13 de febrero.

Commentary Magazine

$20131^{\circ}$ de junio 
Cuttita, Paolo

2006 "Ponts and Lines: A Topography of Borders in the Global Space", Ephemera: Theory \& Politics in Organization, vol. 6, no. 1, en <http:/ / www.ephemeraweb. org/journal/6-1/6-1cuttitta.pdf>.

DEPARTMENT OF STATE

2011 "America's Pacific Century. Remarks", en <http:/ / www.state.gov/ secretary/ rm/2011/11/176999.htm>, 10 de noviembre.

Fergusson, IAN y Bruce VAughn

2009 “The Trans-Pacific Strategic Economic Partnership Agreement”, Congressional Research Service, en <http: / / www.sice.oas.org/TPD/TPP/Studies / USCongress2009_e.pdf $>$, diciembre.

FOREIGN AFFAIRS

2013 julio-agosto.

HalPer, Stefan y Jonathan Clarke

2007 The Silence of the Rational Center. Why American Foreign Policy is Failing, Nueva York, Basic Books.

HunT, MichaEL

1987 Ideology and U.S. Foreign Policy, Nueva York, Yale University Press.

JONES, JEFFREY M.

2011 "Obama Approval Rallies Six Points to 52 \% after Bin Laden Death, Gallup", en <http: / / www.gallup.com/poll/147437/ obama-approval-rallies-six-pointsbin-laden-death.aspx $>, 5$ de mayo.

KatZMan, KenNETH

2011 "Afghanistan: Post-Taliban Governance, Security, and U.S. Policy, Congressional Research Service", en <http:/ / www.fas.org/sgp/crs / row / RL30588.pdf>, 21 de octubre.

KenNeth, ANDERSON

2013 "The Case for Drones", Commentary Magazine, junio. 
Kurt Cronin, Audrey

2013 "Why Drones Fail”, Foreign Affairs, vol. 92, no. 4 junio-agosto.

MANN, JAMES

2012 The Obamians, Nueva York, Viking.

Miller, Greg

2011 "U.S. Officials believe Al-Qaeda on Brink of Collapse", The Washington Post, en <http: / / www.washingtonpost.com/world/national-security/al-qaeda-couldcollapse-us-officials-say / 2011 / 07 / 21 / gIQAFu2pbI_story.html?tid=sm_ twitter_washingtonpost $>, 26$ de julio.

Nossel, SuZAnNe

2004 "Smart Power", Foreign Affairs, vol. 83, no. 2, marzo-abril.

NYE, JOSEPH

2011 The Future of Power, Nueva York, PublicAffairs.

2002- "Limits of American Power", Political Science Quarterly, vol. 117, no. 4, 2003 invierno, pp. 545-559.

2002 "The American National Interest and Global Public Goods", International Affairs, vol. 78, no. 2.

OCDE

s.f. $\quad$ "Statistics from A to Z", en <http:/ / www.oecd.org/document/0,3746,en_ 2649_201185_46462759_1_1_1_1,00.html>.

Office of Management and Budget

2011 "Historical Tables. Budget of the U.S. Government, Fiscal Year 2011", en $<$ http: / / www.gpoaccess.gov/ usbudget/fy11/pdf/hist.pdf >.

OfFice of THE Under Secretary of DefEnse

2011 “National Defense Budget Estimates for Fiscal Year 2012", en <http:/ / comptroller. defense.gov/defbudget/fy2012/FY12_Green_Book.pdf>, marzo.

OfFice of the U.S. TRAde Representative

2011 "The United States in the Trans-Pacific Partnership", en <http:/ / www.ustr. gov / about-us / press-office / fact-sheets / 2011 / november / united-statestrans-pacific-partnership $>$. 
Parent, Joseph M. y Paul MacDonald

2011 "The Wisdom of Retrenchment", Foreign Affairs, vol. 69, no. 6 (noviembrediciembre).

Perlo-Freeman, Sam et al.

2011 "Military Expenditure, SIPRI Yearbook 2011. Armaments, Disarmament and International Security", Stockholm International Peace Research Institute, en <http: / / www.sipri.org/yearbook/2011 / files/SIPRIYB1104-04A-04B.pdf>.

Putin, VLADIMIR

2013 "A Plea for Caution from Rusia", The New York Times, 11 de septiembre.

RoHDE, DAviD

2012 "The Obama Doctrine", Foreign Policy, en <http: / www.foreignpolicy.com/ articles / 2012/02/27/the_obama_doctrine?page $=0,0>$, marzo-abril.

United Nations Development Programme

2011 "Human Development Report 2011. Sustainability and Equity: A Better Future for All", en <http: / / hdr.undp.org/en/reports/global/hdr2011/download/>.

Velasco Grajales, Jesús

2010 Neoconservatives in U.S. Foreign Policy under Ronald Reagan and George W. Bush. Voices behind the Throne, Washington, D.C., Woodrow Wilson Center Press/The Johns Hopkins University Press.

Walt, Stephen M., Thomas L. Friedman y Michael Mandelbaum

2011 "The Myth of American Exceptionalism", Foreign Policy, noviembre, pp. 72-78.

White House, The

s.f. "Defense Guiding Principles", en <http:/ / www.whitehouse.gov/issues / defense>.

2011 "Remarks by the President on the Middle East and North Africa", en <http:/ / www.whitehouse.gov/the-press-office/2011/05/19/ remarks-president -middle-east-and-north-africa>, 19 de mayo.

2010a "National Security Strategy", en <http: / / www.whitehouse.gov/ sites/ default/ files / rss_viewer/national_security_strategy.pdf $>$, mayo.

2010b "Remarks by the President in Address to the Nation on the End of Combat Operations in Iraq", en <http:/ / www.whitehouse.gov/the-press-office/2010 / 
08/31 / remarks-president-address-nation-end-combat-operations-iraq>, 31 de agosto.

2009a "Remarks by the President on a New Beginning", en <http:/ / www.whitehouse. gov / the-press-office / remarks-president-cairo-university-6-04-09>, 4 de junio.

2009b "Remarks of President Barack Obama. Responsibly Ending the War in Iraq", en <http://www.whitehouse.gov/the_press_office/Remarks-of-PresidentBarack-Obama-Responsibly-Ending-the-War-in-Iraq/>, 27 de febrero.

WOODWARD, BOB

2010 Obama's Wars, Nueva York, Simon and Schuster.

2004 Plan of Attack, Nueva York, Simon and Schuster.

2000 Bush at War, Nueva York, Simon and Schuster.

WORLD BANK, THE

S.A. Data, en <http: / / data.worldbank.org/>. 
